\title{
Heterogeneous Kinetic Study for Esterification of Acetic Acid with Ethanol
}

\author{
E.C.L De Silva, B.A.N.N. Bamunusingha and M.Y. Gunasekera
}

\begin{abstract}
The esterification of acetic acid with ethanol has been investigated in the presence of a low cost ion exchange resin mainly used in the water treatment industry namely Trilite SCR-B as a heterogeneous catalyst. Internal mass transfer limitations have proven to be negligible using analysis based on Thieles modulus. The results of the experimental runs done by varying stirrer speeds of the reactor showed the absence of external mass transfer limitations. The kinetic experimental results were correlated with the heterogeneous kinetic models Langmuir-Hinshelwood (LH), Eley-Rideal (ER) and pseudo homogeneous kinetic model. The LH model was found to be correlating the experimental results better than the other two models. Reaction and adsorption constants were further evaluated within the temperature range between $335 \mathrm{~K}$ and $355 \mathrm{~K}$, with 1:1 ethanol to acetic moles ratio under a catalyst loading of $50 \mathrm{~g} / \mathrm{L}$. The study results of adsorption of both water and ethanol on to the cation exchange resin surface were found to be exothermic under the tested conditions. Furthermore, effect of concentration of each reactant on the reaction rate was examined using initial reaction rate measurements.
\end{abstract}

Keywords: Heterogeneous catalysis, esterification kinetics, cation exchange resin,

\section{Introduction}

Ethyl Acetate is a valuable organic ester which is highly applied in perfumery, paint and coating industry, pharmaceutical industry and flavours in food industry [1], [2]. The commercial process of manufacturing ethyl acetate is based on the reaction of esterification between ethanol and acetic acid under an acid catalyst.

$$
\mathrm{CH}_{3} \mathrm{COOH}+\mathrm{C}_{2} \mathrm{H}_{5} \mathrm{OH} \rightarrow \mathrm{CH}_{3} \mathrm{COOC}_{2} \mathrm{H}_{5}+\mathrm{H}_{2} \mathrm{O}
$$

However, several drawbacks are met in using homogeneous acid catalysts for the above reaction. Corrosion problems driven by liquid catalyst, huge energy consumption for separation of product from catalyst, waste of catalyst and lack of purity in the product represent these drawbacks [3].

Use of heterogeneous catalysts has been a major interest as an alternative type of catalyst. Ismail et al. [3] have studied the applicability of ion exchange resin, Amberlyst-15 for the same reaction. The results have been successfully fitted with a model proposed by them which is similar to the ER model. The study of Hangx et al. [4] has shown an evaluation of the same reaction with the cation exchange resin Purolite CT179 and their results have proven that forward reaction rate is proportional to the $1.5^{\text {th }}$ power of the acetic acid concentration. Furthermore, ion exchange resins, Amberlyst-
15, D001, D002 and NKC have been successfully applied to catalyse the esterification of lactic acid with ethanol by Zhang et al. [5]. Their work has generated experimental results that obey the LH mechanism. Further, they have proven that the activation energies for the catalysts D002 and NKC under the tested conditions lie between $51 \mathrm{~kJ} / \mathrm{mol}$ and $53 \mathrm{~kJ} / \mathrm{mol}$.

The resins used in above studies are costly making it a significant issue in scaling up the findings of those studies. Further they are not available in the local market. However ion exchange resins used in the industrial water treatment are available at low costs. Performance data on the use of low cost ion exchange resins used in the water treatment industry as a catalyst in the esterification of acetic acid with ethanol are not available in literature. Therefore evaluation of the performance and kinetic data for low cost cation exchange resins has been a requirement.

E.C.L De Silva, Undergraduate Student B.Sc. Eng. Degree Course, Chemical \& Process Engineering Department, University of Moratuwa, Sri Lanka

Mrs. B.A.N.N. Bamunusingha, B.Sc. Eng. (Hons)

(Moratuwa), Research Student, Chemical \& Process Engineering Department, University of Moratuwa, Sri Lanka

Eng. (Dr.) (Ms) M.Y. Gunasekera, B.Sc. Eng. (Hons) (Moratuwa), MIE (Sri Lanka), M.Eng. (Moratuwa), PhD (UK), Senior Lecturer, Chemical \& Process Engineering Department, University of Moratuwa, Sri Lanka 
In the present study, esterification reaction between acetic acid and ethanol, under the locally available low cost cation exchange resin Trilite SCR-B which is commonly used in water purification is investigated. This resin which is commercially available in $\mathrm{Na}^{+}$form is treated to obtain an acidic cation exchange resin of $\mathrm{H}^{+}$ form before being used in this study. The experimental kinetic data are correlated using Langmuir-Hinshelwood model, Eley-Rideal model and pseudo homogeneous kinetic model in order to find which one describes the reaction kinetics better. Effect of initial ethanol and acetic acid reactant concentrations on the initial rate of reaction has been studied in order to experimentally investigate the tendency of adsorption of reactants onto the catalyst surface. Adsorption constants and adsorption enthalpies were also studied.

\section{Materials and Method}

\subsection{Materials}

Analytical grade Acetic acid of purity 99.8\% $(w / w)$ and analytical grade ethanol of purity $99.8 \%(\mathrm{w} / \mathrm{w})$ were used as the reactants for each of the experimental runs. Cation exchange resin, Trilite SCR-B which is available in the local market was used as the heterogeneous catalyst for the reaction. Properties of the ion exchange resin used are summarized in Table-1. $\mathrm{HCl}$ of $35 \%(\mathrm{w} / \mathrm{w})$ and de-ionized water were used for catalyst preparation. The $\mathrm{NaOH}$ solution used in titrations was prepared using $98 \% \mathrm{NaOH}$ pellets. All the solutions were prepared using distilled water.

Table 1 - Properties of the ion exchange resin Trilite SCR-B

\begin{tabular}{|c|c|}
\hline Physical Property & Description \\
\hline Physical form & $\begin{array}{c}\text { Brown spherical } \\
\text { grain }\end{array}$ \\
\hline Ionic form as shipped & $\mathrm{Na}^{+}$ \\
\hline Moisture content (\%) & $43-50$ \\
\hline Grain size range & $(0.3-1.2) \geq 95 \%$ \\
\hline Shipping density (g/l) & 842 \\
\hline $\begin{array}{c}\text { Maximum operating } \\
\text { temperature (K) }\end{array}$ & 393 \\
\hline
\end{tabular}

\subsection{Apparatus}

The reaction was carried out in a three necked round bottom flask of $500 \mathrm{ml}$ which was immersed in a water bath. A temperature controllable magnetic stirrer hot plate was used to heat the water bath in order to maintain the required temperature in the flask. The reaction mixture was stirred using a magnetic stirrer to ensure the homogeneity. One neck of the flask was connected to a total reflux condenser to prevent the loss of material during the reaction time while another neck was used to insert the thermometer with an accuracy of $\pm 1 \mathrm{~K}$. The third neck was used as the sampling point.

\subsection{Procedure}

\subsubsection{Catalyst Preparation}

Cation exchange resin was soaked in 35\% $(\mathrm{w} / \mathrm{w}) \mathrm{HCl}$ for 30 minutes, and then washed several times with de-ionized water. Subsequently, the resin was dried in the oven at a temperature of $363 \mathrm{~K}$ and was stored in the desiccator until it's been used. $\mathrm{HCl}$ concentration for soaking the resin, and resin drying time were selected based on the results of the study of Bamunusingha et al [6].

\subsubsection{Reaction kinetic measurements}

Acetic acid was charged into the three necked flask and the measured amount of dried catalyst was added to the same reactor. The mixture of acetic acid with catalyst was heated up to the desired temperature. Other reactant, ethanol, preheated to the same temperature was then poured into the reactor and this instant was taken as the time zero for the run. The mixture was stirred continuously with the magnetic stirrer and samples were withdrawn in regular intervals. Withdrawn samples were instantly poured into distilled, chilled water to freeze the reaction. These samples were quickly titrated with standardized $1 \mathrm{M} \mathrm{NaOH}$ using phenolphthalein as the indicator in order to determine the amount of unconverted acetic acid in the sample.

\section{Results and Discussion}

\subsection{Internal diffusion}

Overall rate of heterogeneous catalytic reactions are influenced not only by the reaction kinetics which determines the surface reaction rate, but also by effects of adsorption, desorption, internal diffusion and external diffusion of reactants and products [7]. From these factors, influence by internal diffusion for the experimental conditions can be evaluated using theoretical analysis and logical reasoning based on the Thieles' modulus and effectiveness factor. 
Thieles' modulus for a spherical catalyst pellet catalyzing a first order reaction can be given by Eq. (1) [8].

$$
\varphi_{s}=\frac{r_{s}}{3} \sqrt{\frac{k_{1} \rho_{p}}{D_{e}}}
$$

Thieles' modulus is denoted by $\varphi_{s}$ here and, $r_{s}$ and $k_{1}$ represent radius of spherical particle and forward rate constant, respectively. Effective diffusion coefficient is given by $\mathrm{D}_{\mathrm{e}}$ and, $\rho_{p}$ is the density of solid catalyst particle. Forward rate constant parameter in Eq. (1) can be eliminated using measured reaction rate $\left(r_{p}\right)$ and concentration within the reaction medium $\left(\mathrm{C}_{\mathrm{S}}\right)$ expressed according to first order kinetics. This results in Eq. (2) which expresses Thieles' modulus for a first order reaction [8].

$$
\varphi_{s}=\frac{r_{s}}{3} \sqrt{\frac{r_{p} \rho_{p}}{C_{s} D_{e}}}
$$

Further, the ratio between actual reaction rate for the whole pellet to the rate evaluated at outer surface conditions identified as effectiveness factor tends to reach unity as the mass transfer resistances are reduced. Furthermore, the relationship curves of effectiveness factor against Thieles' modulus for different orders of reactions have been observed to be almost overlapping at very low Thieles' modulus values [8]. Therefore if the Thieles' modulus value calculated using Eq. (2) is reasonably low for a higher order reaction, this calculated value confirms its validity though Eq. (2) is applicable for first order reactions at first place. This conclusion further facilitates the use of this calculated Thieles' modulus in effectiveness factor vs. Thieles' modulus relationship curves.

In the experimental runs, it was assumed that no significant difference occurs in ratio $r_{P} / C_{S}$ throughout the reaction time. Therefore initial rate of reaction and concentrations were used in Eq. (2). Initial reaction rate was determined by Eq. (3), using graphical method to find the derivative, $d X / d t$ in which $X$ and $t$ represent fractional decomposition and time respectively.

$$
r_{P}=\frac{c_{0}}{W}\left(\frac{d X}{d t}\right)
$$

$\mathrm{W}$ and $\mathrm{C}_{0}$ are catalyst loading and initial concentration of reference species respectively. Further using dry density of the resin catalyst as $\rho_{p}$ in Eq. (2), the Thieles' modulus for the catalyst particle in this study is yielded as 0.51 . This is a lower value enough to result in an effectiveness factor closer to unity according to the effectiveness factor vs. Thieles' modulus relationship curves [8]. Therefore effects of internal mass transfer resistances during the reaction can be considered as not affecting the overall rate of reaction significantly.

\subsection{External diffusion}

Effect of external diffusion on the overall rate of reaction was evaluated. Diffusion of reactants and products in the bulk fluid outside the catalyst particle directly depends on the turbulence within the reaction mixture. Two experimental runs were carried out with the same setup at $335 \mathrm{~K}$, using a catalyst loading of $50 \mathrm{~g} / \mathrm{L}$, with resin prepared under same conditions, at two different stirrer speeds. In the heating magnetic stirrer named AREX manufactured by VELP Scientifica, 3.5 and 5.5 units of the scale of 10 units were used respectively as the lower speed and the higher speed. Kinetic data were observed for about 45 minutes and the observations have been plotted in Figure 1. The Mean Residual Deviation (MRD \%) between the observation points of the two stirrer speed runs was calculated to be $4.7 \%$. This indicates that external mass transfer effects are negligible within this range of stirrer speeds. Therefore all the kinetic experimental runs of this study were carried out maintaining the stirrer speed at 3.5 to ensure the absence of external mass transfer resistances.

\subsection{Kinetic modelling}

Heterogeneous reaction has been described with many models depending on the characteristics of the reactants and the catalyst particle. Esterification reaction carried out in this study using cation exchange resin, Trilite SCR-B catalyst has been modeled using different kinetic models in this section and the best mechanism to correlate the kinetic data has been evaluated.

\subsubsection{Kinetic models}

Langmuir-Hinshelwood (LH) model is found to be the most applicable model in the cases of many heterogeneous catalysts used for this kind of esterification reactions [5]. This kinetic model assumes both acetic and the ethanol molecules to be adsorbed on to the catalyst surface prior to the surface reaction. Final stage of the mechanism involves desorption of the two products, ethyl acetate and water.

In Eley-Rideal (ER) model, it is assumed that only one reactant adsorbs on to the catalyst surface while the other reactant is in the bulk solution during the reaction. According to this mechanism, two of the four species which 
participate in the reaction are susceptible to be more likely to undergo with sorption than other two species. Therefore two species are accounted for sorption effects in ER models' kinetic expression. Literature shows that water and ethanol are more likely to be involved in sorption effects in this mechanism [9].

Third kinetic model in the interest is the pseudo homogeneous model. This mechanism treats the catalyst particles holding positive charged sites, as large cations dissolved in the reaction mixture [10].

Model equations for LH model, ER model and pseudo homogeneous model could be written as follows [11].

(i) LH model

$$
r=k \frac{C_{A c} C_{E}-\frac{C_{E A} C_{W}}{K_{e q}}}{\left(1+k_{w} C_{W}+k_{E} C_{E}+k_{A c} C_{A c}+k_{E A} C_{E A}\right)^{2}}
$$

(ii) E-R model

$$
r=k \frac{C_{A c} C_{E}-\frac{C_{E A} C_{W}}{K_{e q}}}{\left(1+k_{w} C_{W}+k_{E} C_{E}\right)}
$$

(iii) Pseudo homogeneous model

$$
\begin{aligned}
& r=k\left[C_{A c} C_{E}-\frac{C_{E A} C_{W}}{K_{e q}}\right] \\
& k_{i}=\frac{c_{i-s}}{C_{i} c_{s}} \quad, \quad K_{e q}=\left(\frac{C_{E A} \times C_{W}}{C_{A c} \times C_{E}}\right)_{e q}
\end{aligned}
$$

In above equations, $\mathrm{k}$ represents the reaction constant and $k_{i}$ represents the adsorption coefficients. Parameters $c_{s}$ and $c_{i-s}$ denote the concentration of vacant cites on catalyst surface and the concentration of component $i$ at the catalyst surface respectively. Concentrations in bulk medium are expressed by $\mathrm{C}$ together with subscripts Ac, E, EA and W for acetic acid, ethanol, ethyl acetate and water respectively.

\subsubsection{Determination of model parameters}

Kinetic data of the experimental run at temperature $335 \mathrm{~K}$ with a catalyst loading of $50 \mathrm{~g} / \mathrm{l}$, were used in correlating the three kinetic models in order to compare the suitability of the three different models to correlate kinetic data.

The results of the studies of Delgado et al. [9] and Altiokka et al. [12] indicate that there is no significant error in using concentrations in model equations instead of activities. Therefore modeling was done in terms of measured concentration values directly instead of using activity coefficients which give mathematical complexity. Instantaneously measured acetic acid concentrations were used with relevant stoicheometric coefficients to determine the instantaneous concentrations of other three species. Equilibrium constant was determined using the concentrations of the four species at

\begin{tabular}{|c|c|c|c|}
\hline Model & $\begin{array}{c}\text { Parameters } \\
*\end{array}$ & Value & $\begin{array}{l}\text { MRD } \\
(\%)\end{array}$ \\
\hline LH model & $\begin{array}{l}\mathrm{k}\left(\mathrm{l}^{2} \mathrm{~g}^{-1} \mathrm{~mol}^{-}\right. \\
\mathrm{k}_{\mathrm{W}}\left(1 \mathrm{~mol}^{-1}\right) \\
\mathrm{k}_{\mathrm{E}}\left(1 \mathrm{~mol}^{-1}\right)\end{array}$ & $\begin{array}{l}1.7 x \\
4.9 x \\
1.8 x \\
10^{-1}\end{array}$ & $5.08 \%$ \\
\hline ER model & $\begin{array}{l}\mathrm{k}\left(\mathrm{l}^{2} \mathrm{~g}^{-1} \mathrm{~mol}^{-}\right. \\
\mathrm{k}_{\mathrm{W}}\left(1 \mathrm{~mol}^{-1}\right) \\
\mathrm{k}_{\mathrm{E}}\left(1 \mathrm{~mol}^{-1}\right)\end{array}$ & $\begin{array}{c}8.7 x \\
0 \\
1.3 x \\
10^{-1}\end{array}$ & $6.62 \%$ \\
\hline $\begin{array}{c}\text { Pseudo } \\
\text { homogeneou } \\
\text { s model }\end{array}$ & $\begin{array}{l}\mathrm{k}\left(\mathrm{l}^{2} \mathrm{~g}^{-1} \mathrm{~mol}^{-}\right. \\
\left.{ }^{1} \mathrm{~min}^{-1}\right)\end{array}$ & $\begin{array}{c}1.03 x \\
10^{-4}\end{array}$ & $14.98 \%$ \\
\hline
\end{tabular}
equilibrium.

Table 2 - Estimated model parameters for different kinetic models at $335 \mathrm{~K}$

*rate constant (k); adsorption coefficient of water $\left(\mathrm{k}_{\mathrm{W}}\right)$; adsorption coefficient of ethanol $\left(\mathrm{k}_{\mathrm{E}}\right)$

Parameter estimation includes the estimation of reaction constant and adsorption coefficients. It was assumed that the adsorption of the molecules was competitive on the same active site, and only those having the strongest adsorption were taken into account in simplified mechanisms. It is found in the literature that water and ethanol are having superior adsorptivity on similar resin catalysts compared to acetic acid and ethyl acetate [9]. Therefore to reduce the complexity of regression analysis in the case of LH model, adsorption coefficients of ethyl acetate and acetic acid were assumed to be negligible [5]. The modified version of LH model could be illustrated as in Eq. (7) with same notifications as in Eq. (4).

$$
r=k \frac{C_{A c} C_{E}-\frac{C_{E A} C_{W}}{K_{e q}}}{\left(1+k_{W} C_{W}+k_{E} C_{E}\right)^{2}}
$$


Regression of the model parameters was carried out by minimizing the sum of residual squares (SRS) as shown in Eq. (8). A user-built function was used for this purpose in Matlab environment. This function was designed to iterate the model over a wide range of combinations of values for rate constant $(\mathrm{k})$ and adsorption coefficients of water and ethanol $\left(\mathrm{k}_{\mathrm{W}}, \mathrm{k}_{\mathrm{E}}\right)$. Each of the iterations integrates the model equation numerically by 4 th order Runge-Kutta method to determine the fractional decompositions at ten observation time points followed by a determination of sum of residual squares of decompositions at these ten observation points. Best combination of parameters which minimized the SRS was taken as the optimum curve fit and the accuracy of the fit was evaluated by means of mean relative deviation (MRD) of observation points using Eq. (9).

$$
\begin{gathered}
S R S=\sum_{N}\left(r_{\text {exp }}-r_{\text {calc }}\right)^{2} \\
M R D=\frac{1}{N}\left(\sum_{N}\left|\frac{X_{\text {calc }}-X_{\text {exp }}}{X_{\text {exp }}}\right|\right) \times 100 \%
\end{gathered}
$$

In Eq. (8) \& (9), N, r and $X$ are number of observation points, reaction rate and fractional decomposition respectively. Subscripts 'exp' and 'calc' indicate experimental and calculated values.

Summary of the parameter values for all three models is given in Table 2. Results indicate that LH model can correlate the kinetic data of this heterogeneous catalytic reaction well with a MRD value of $5.08 \%$ which is the minimum compared with that of the rest of the two models. Similar MRD\% values have been observed by other researchers for similar reactions. For esterification of lactic acid with methanol using D002 and NHK cation exchange resins, MRD values around $4 \%$ have been observed by Zhang et al. [5]. However, ER model is also having a low mean relative deviation value which makes it also applicable to correlate kinetic data of Trilite SCR-B resin. It is interesting to notice that adsorption coefficient of water is zero in the case of ER model. This indicates that sorption of water on catalyst occurs faster than that of ethanol during the reaction. Results of sorption experiments done by Delgado et al. [9] have also revealed the same information. They have studied the Amberlyst-15 ion exchange resin catalyst with lactic and ethanol reaction. Their study results also have been correlated effectively with LH model.

\subsection{Effect of reaction temperature}

\subsubsection{Model parameters}

Effect of temperature on the esterification reaction has been studied over a range of temperatures. Different experimental runs were carried out using the same procedure with temperatures of $335 \mathrm{~K}, 345 \mathrm{~K}$ and $355 \mathrm{~K}$. As shown in sections 2.1 and 2.2, the internal and external mass transfer limitations were assumed to be negligible. Further, since LH model was found to be the best in correlating the kinetic data of this reaction at $335 \mathrm{~K}$, kinetic data at each of the other temperatures were also modelled directly using LH model given in Eq. (7). The reaction constants, adsorption coefficients, SRS and MRD values for each temperature are summarized in Table 3. Figure 1 illustrates the modelled curves of fractional decomposition against time compared with the experimental values.

Table 3 - Estimated model parameters at different temperatures using LH model

\begin{tabular}{cccccc}
\hline $\begin{array}{c}\text { Temper } \\
\text { ature }\end{array}$ & $\begin{array}{c}\mathbf{k} \mathbf{( 1}^{\mathbf{2}} \mathbf{g}^{-1} \\
\mathbf{m o l}^{-1} \\
\left.\mathbf{m i n}^{-1}\right)^{*}\end{array}$ & $\begin{array}{c}\mathbf{k w}(\mathbf{l} \\
\left.\mathbf{m o l}^{-1}\right)^{*}\end{array}$ & $\begin{array}{c}\mathbf{k E}_{\mathbf{~}} \mathbf{l} \\
\left.\mathbf{m o l}^{-1}\right)^{*}\end{array}$ & SRS & $\begin{array}{r}\mathbf{M} \\
\mathbf{R D} \\
\mathbf{( \% )}\end{array}$ \\
\hline $335 \mathrm{~K}$ & $1.7 \times 10^{-4}$ & $4.9 \times 10^{-2}$ & $1.8 \times 10^{-1}$ & $1.65 \times 10^{-3}$ & $5.08 \%$ \\
$345 \mathrm{~K}$ & $5.0 \times 10^{-4}$ & $3.2 \times 10^{-2}$ & $1.3 \times 10^{-1}$ & $5.65 \times 10^{-4}$ & $2.96 \%$ \\
$355 \mathrm{~K}$ & $9.0 \times 10^{-4}$ & $1.4 \times 10^{-2}$ & $1.6 \times 10^{-1}$ & $2.76 \times 10^{-4}$ & $1.24 \%$
\end{tabular}

${ }^{*}$ rate constant (k); adsorption coefficient of water $\left(\mathrm{k}_{\mathrm{W}}\right)$; adsorption coefficient of ethanol $\left(\mathrm{k}_{\mathrm{E}}\right)$.

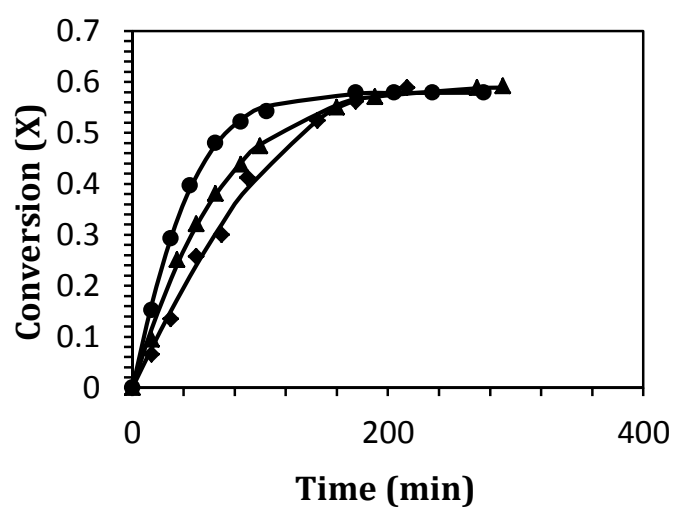

Figure 1 - Experimental points of conversion vs. time at temperatures $335 \mathrm{~K}(\bullet), 345 \mathrm{~K}(\Delta)$, 355K (•) with Acetic:Ethanol=1:1, Catalyst loading $50 \mathrm{~g} / l$, solid line $(-)$ denotes calculated curve using LH model.

According to the study of Bamunusingha et al. [6], it has been found that for the same reaction conducted under the same conditions and cation exchange resin requires an activation energy of $42.8 \mathrm{~kJ} / \mathrm{mol}$. This activation energy had been calculated based on the pseudo homogeneous model and therefore the 
involvement of adsorption of the molecules had been neglected.

\subsubsection{Adsorption enthalpies}

Variation of adsorption coefficients $\left(\mathrm{k}_{\mathrm{i}}\right)$ against temperature can be described using Van't Hoff law given in Eq. (10) [13]. Using the adsorption coefficient results determined from LH model for different temperatures as shown in table 3 and Eq. (10), the Van't Hoff's plots have been obtained (Figure $2 \& 3$ ). Adsorption enthalpy results determined using the plots are summarized in Table 4.

Table 4 - Adsorption Enthalpy Values

\begin{tabular}{lcc}
\hline & Water & Ethanol \\
\hline$\Delta \mathrm{H}\left(\mathrm{kJ} \mathrm{mol}^{-1}\right)$ & -61.6 & -5.8 \\
\hline
\end{tabular}

$$
\ln \left(k_{i}\right)=\ln \left(k_{i}{ }^{0}\right)-\frac{\Delta H_{i}}{R T}
$$

In Eq. (10), $\mathrm{T}$ and $\mathrm{R}$ represent absolute temperature and the universal gas constant respectively. Adsorption enthalpy of the respective species is given by $\Delta \mathrm{H}_{\mathrm{i}}$ in the Van't Hoffs' equation. Subscript ' $i$ ' may refer to water or ethanol.

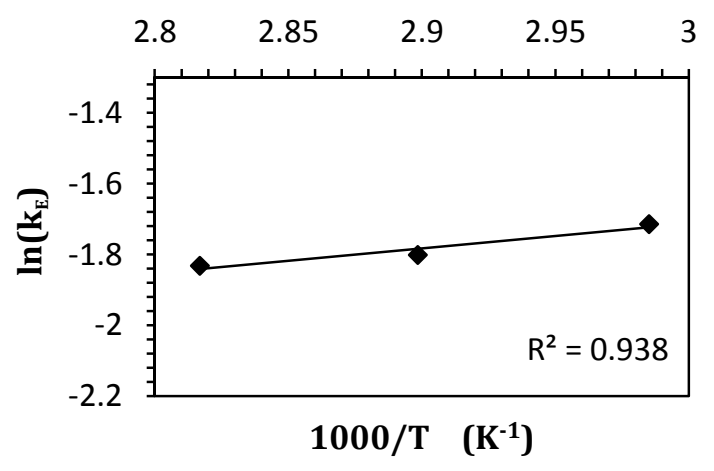

Figure 2 - Van't Hoff plot of adsorption coefficients of ethanol, $R^{2}=0.938$.

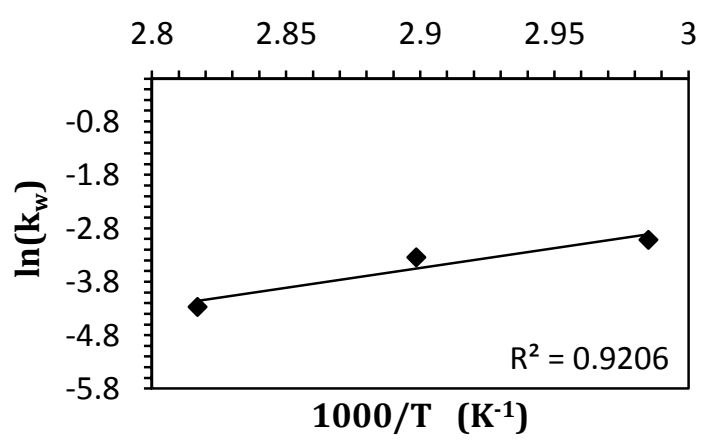

Figure 3 - Van't Hoff plot of adsorption coefficients of water $R^{2}=0.920$.
Zhang et al. [5] have obtained adsorption enthalpy values $-6.42 \mathrm{~kJ} / \mathrm{mol}$ and $-21.33 \mathrm{~kJ} / \mathrm{mol}$ for water and ethanol, respectively in their study using the resin D002 which is also a strongly acidic cation exchange resin. Although both of the adsorption enthalpies of water and ethanol on to the catalyst Trilite SCR-B are exothermic, unlike in Zhangs' [5] results, the adsorption of water seems to be releasing a higher amount of energy than ethanol in the current study. This suggests that adsorbed water molecule becomes more stable than the ethanol molecule adsorbed onto the catalyst surface. It can be explained by hydrophilic characteristics of this cation exchange resin which is commonly used in water purification.

\subsection{Effect of reactant concentrations}

Experimental methods were carried out to investigate the effect of reactant concentrations on rate of reaction. From the two reactants Acetic acid and Ethanol, each one was kept at a constant concentration value of $6 \mathrm{~mol} / \mathrm{L}$ for several experimental runs while other reactant concentration was varied. The solvent nheptane was used to maintain the total reaction volume at $100 \mathrm{ml}$ in each run and temperature was maintained at $336 \pm 1 \mathrm{~K}$. Initial reaction rates were determined by the gradient of the tangent drawn to the decomposition curve [7]. Variation of initial reaction rate against Acetic acid and ethanol concentrations are shown in Figure (4) and (5) respectively.

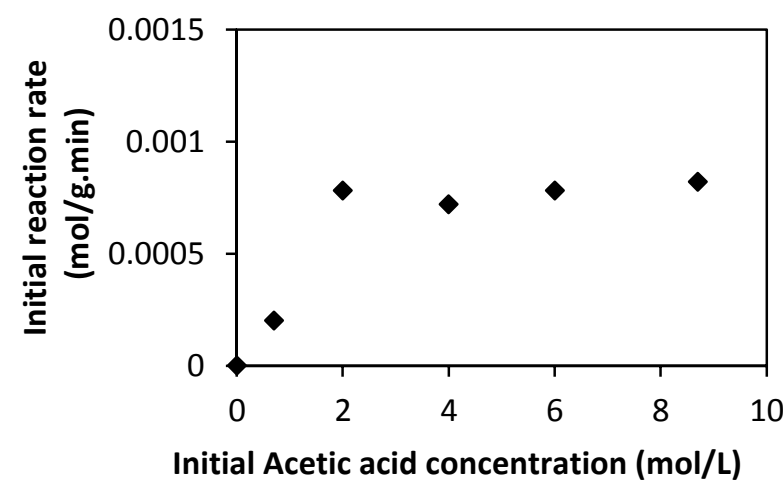

Figure 4 - Effect of Acetic acid concentration on initial reaction rate: $\left(C_{E t h a n o l, 0}=6 \mathrm{~mol} / \mathrm{L}\right.$, $\mathrm{C}_{\text {water }}=\mathrm{C}_{\mathrm{EA}}=0 \mathrm{~mol} / \mathrm{L}$, Temperature: $337 \mathrm{~K}$, Catalyst loading: $50 \mathrm{~g} / \mathrm{L}$ ) 


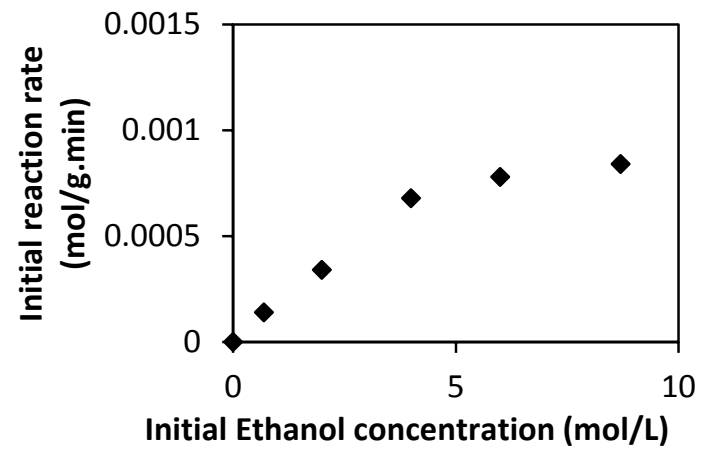

Figure 5 - Effect of Ethanol concentration on initial reaction rate: $\left(C_{\text {Acetic, } 0}=6 \mathrm{~mol} / \mathrm{L} ; C_{\text {water }}=\right.$ $\mathrm{C}_{\mathrm{EA}}=0 \mathrm{~mol} / \mathrm{L}$; Temperature: $337 \mathrm{~K}$; Catalyst loading: $50 \mathrm{~g} / \mathrm{L}$ )

The reaction rate tends to be not affected significantly by any of the reactant concentrations within the range of $4 \mathrm{~mol} / \mathrm{L}$ and $8.65 \mathrm{~mol} / \mathrm{L}$. Since these measured concentrations supposed to be pertaining to the bulk fluid, it can be explained that reaction rate is mostly controlled by the adsorbed reactant concentrations of both reactants on the catalyst surface within this concentration range.

The kinetics data presented in section 3 above are essential information required by engineers who are involved in designing reactors. The kinetics involved in a chemical reaction is independent of the scale of the reaction operation carried out [7]. Therefore, the LH model with its' determined kinetic data in this work could be used in large scale engineering applications such as designing of industrial esterification reactors for producing ethyl acetate, using reactants acetic acid and ethanol with the catalyst Trilite SCR-B.

\section{Conclusions}

This study investigated the heterogeneous kinetics of esterification of acetic acid with ethanol using cation exchange resin, Trilite SCR-B as the catalyst. The reaction is kinetically controlled in which the overall rate of reaction is neither affected by internal mass transfer resistances nor by external mass transfer resistances. The heterogeneous reaction mechanism is more close to the LangmuirHinshelwood model among all the three kinetic models used where Eley-Rideal model and pseudo homogeneous model being the other two models. Rate of reaction is neither significantly affected by any of the two reactant concentrations in the bulk phase for the range of reactant concentrations observed in the study. In relation with the LangmuirHinshelwood model, adsorption of both water and ethanol on to the cation exchange resin Trilite SCR-B, are exothermic while water molecules adsorbed on to the catalyst surface showed more stability than ethanol molecules.

\section{References}

1. Dutia, P., "Ethyl Acetate: A Techno-Commercial Profile", Chemical weekly, August, 2010.

2. Bijay, N. P., Hiren, C.M., "Ethyl Acetate: Properties, Production Process and Applications A Review", International Journal of Current Research and Review, pp. 23-40, 2011.

3. Ismail, S. K., Baris, Z. B., Umur, D., "Esterification of Acetic Acid with Ethanol Catalyzed by an Acidic Ion Exchange Resin", Turk J Engin Environ Sci 25 (2001), pp. 569-577, 2000.

4. Hangx, G., Kwant, G., Maessen, H., Markusse, P., Urseanu, I., "Reaction Kinetics of the Esterification of Ethanol and Acetic Acid towards Ethyl Acetate", Intelligent Column Internals for Reactive Separations, August, 2001.

5. Zhang, Y., Ma, L., Yang, J., "Kinetics of esterification of lactic acid with ethanol catalyzed by cation-exchange resins", Reactive $\mathcal{E}$ Functional Polymers, pp. 101-114, 2004

6. Bamunusingha, B. A. N. N., Gunasekera, M. Y., "Production of Ethyl Acetate using Catalytic Reaction" Annual Transactions of IESL , pp. 370378,2011

7. Fogler, H. S., Elements of Chemical Reaction Engineering, 4th ed., New Delhi, 2006.

8. Smith, J. M., Chemical Engineering Kinetic, $2^{\text {nd }}$ ed.,New York, 1970, p. 427-435.

9. Delgado, P., Sanz, M. T., Beltran, S., "Kinetic Study for Esterification of Lactic Acid with Ethanol and Hydrolysis of Ethyl Lactate using an Ion-Exchange Resin catalyst", Chemical Engineering Journal, pp. 111-118, 2007.

10. Ehteshami, M., Rahimi, N., Eftekhari, A. A., Nasr, M. J., "Kinetic Study of Catalytic Hydrolysis Reaction of Methyl Acetate to Acetic Acid and Methanol", Iranian Journal of Science E Technology, 2006.

11. Schwarzer, R., "Esterification of Acetic Acid with Methanol: A Kinetic Study on Amberlyst 15", A Thesis for Degree of Masters of Engineering, University of Pretoria, Pretoria, 2006.

12. Altiokka, M.R., Citak, A., "Kinetics Study of Esterification of Acetic Acid with Isobutanol in the Presence of Amberlite Catalyst", Applied Catalysis, pp. 141-148, 2003.

13. Benedict, D., Parulekar, S. J., Tsai, S. P., "Esterification of Lactic Acid and Ethanol With/Without Pervaporation", Ind. Eng. Chem. Res, pp. 2282-2291, 2003. 
